\title{
Recent developments towards closing the gap in osteoporosis management
}

\author{
Yasser El Miedany
}

\begin{abstract}
Background: A fracture that occurs in people with low bone mass in the setting of minimal trauma-such as a fall from standing height-meets the criteria for the clinical diagnosis of osteoporosis and qualifies this particular individual for being at high risk of further fractures, particularly in the first 2 years after the index fracture. Therefore, it is vital to identify those individuals at very high and high fracture risk with the potential of instantly starting osteoporosis therapy.

Main body: Currently, there are unmet needs in the management of bone fragility and fracture prevention. Therefore, re-stratification of the people according to their risk of fracture, and, also, identify what is and is not achievable using different osteoporosis therapies, represent a major step forward. In 2020, the dichotomisation of high risk into high and very high-risk categories, which represent a new concept in osteoporosis assessment, was published by the IOF and the ESCEO. This coincided with proliferation of the available therapies with different modes of action and new therapeutic targets for treating osteoporosis. Fear of complications, even though rare, associated with long-term bisphosphonates and the positive impact of osteoanabolic agents on fracture reduction and bone quality, have changed the prescribing patterns and paved the way for sequential and combined therapy.

Conclusion: The incorporation of recent concepts in osteoporosis and the development of new interventional thresholds have positive implication on strategies for osteoporotic patients' diagnosis and management.
\end{abstract}

Keywords: Osteoporosis, FRAX, BMD, Bisphosphonates, Anabolic therapy, Sequential, Combination therapy, Anabolic window

\section{Background}

As the main target of osteoporosis treatment is to reduce the risk of sustaining fragility fractures, there has been a significant shift in the paradigm of osteoporosis assessment and management $[1,2]$. Historically, the cornerstone of the fragility fracture risk assessment has been based on measurement of bone mineral density (BMD). In the absence of a true gold standard, the WHO has defined osteoporosis as a BMD that lies 2.5 standard deviations or more below the average value for young healthy women (a $T$-score of $\leq-2.5 \mathrm{SD})[3,4]$. Although the osteoporosis diagnostic criteria set by the WHO were primarily meant for descriptive epidemiology, later, it was adopted in clinical and medications trials as

Correspondence: miedanycrd@yahoo.com

King's College, London, England

\section{Springer Open}

(c) The Author(s). 2021 Open Access This article is licensed under a Creative Commons Attribution 4.0 International License, which permits use, sharing, adaptation, distribution and reproduction in any medium or format, as long as you give appropriate credit to the original author(s) and the source, provide a link to the Creative Commons licence, and indicate if changes were made. The images or other third party material in this article are included in the article's Creative Commons licence, unless indicated otherwise in a credit line to the material. If material is not included in the article's Creative Commons licence and your intended use is not permitted by statutory regulation or exceeds the permitted use, you will need to obtain permission directly from the copyright holder. To view a copy of this licence, visit http://creativecommons.org/licenses/by/4.0/. 
care algorithm including FRAX and drug targeting or usual primary care for osteoporosis based on opportunistic case finding. Results revealed that the treatment strategy based on BMD (lowest $T$-score of two site assessment) weakens the power of FRAX by excluding some high-risk individuals [8]. On another front, analyses of four phase 3 studies of raloxifene [9], strontium ranelate [10], and teriparatide [11, 12] have elaborated several important implications. First, they alleviate the concern that patients identified based on FRAX clinical risk factors would not respond to medical interventions. In fact, highfracture probabilities estimated by FRAX were correlated to therapeutic efficacy, even when BMD was not implemented to identify the risk. Second, they endorse the concept that medical management should be targeted favourably to men and women at high risk of sustaining fragility fracture(s). Third, as treatments directed to patients with the high-fracture risk probability, has positive impact on the budget; approaching the higher-risk groups can be considered as a cost-effective intervention [13]. Lastly, other studies that assessed the relation between $T$-score and fracture risk revealed that any given $T$-score threshold has a different significance at different ages [14-18].

In view of this, it has been suggested that interventions based on BMD thresholds alone, do not optimally identify subjects at high risk of sustaining fragility fractures, and support the rationale for implementing/developing risk assessment tools able to influence osteoporotic patients' management [19-21]. The scope of this article is to discuss the incorporation of recent concepts in osteoporosis, the development of new interventional thresholds and its implication on strategies for osteoporotic patients' diagnosis and management.

\section{Main text}

\section{Case finding strategy}

In 2018, the guidelines for the diagnosis and management of postmenopausal osteoporosis were updated by the International Osteoporosis Foundation (IOF) and the European Society for Clinical and Economic Evaluation of Osteoporosis and Osteoarthritis (ESCEO) [22]. However, to be applicable for use in standard day to day practice and to facilitate the identification and management of individuals at high-fracture risk, these guidelines had to be transformed into practical algorithms. According to IOF and ESCEO recommendations, the fracture risk should be expressed as an "absolute risk". This means the fracture probability over an interval of 10 years [22]. The absolute fracture risk relies on age, life expectancy, and the current risk of fracture. The 10-year period was selected to cover the likely duration of medical management and the time period over which benefits may last or risks occur if osteoporosis therapy was stopped [23].
In concordance with most of the published guidelines, subjects who sustained a prior fragility fracture can be considered for osteoporosis management without the need for further risk assessment, though BMD assessment may be advised particularly in younger people or to monitor medical therapy. Earlier studies revealed that immediately after an index fracture, the risk of a subsequent osteoporotic fracture is particularly acute; and the risk wanes progressively over time [24-29]. This very high risk of fracture and the subsequent further loss of utility occurring instantly after a consequent fracture (termed "imminent risk" attributed to the temporal association), endorse the concept that preventive medical management given as soon as possible after a fragility fracture would help to minimize the risk of a higher number of new fractures and decrease the possible associated morbidity; compared to therapy given later. This new concept of imminent fracture risk supports the rationale for very early intervention immediately after a sentinel fracture and mandates management with therapies that have the most rapid impact on fracture reduction. A further development is the recent demonstration of an extra rapid and greater fracture risk reduction induced by anabolic agents in contrast to that reported with anti-resorptive therapies [30-34]. This represents a potential to revolutionise osteoporosis management strategies, particularly in subjects at very high-fracture risk $[35,36]$. Therefore, in addition to the standard clinical risk factors, it became vital for the case finding process to identify those individuals at very high and high risk of fragility fracture(s).

\section{The concept of very high-fracture risk}

In 2020, the dichotomisation of high risk into high- and very high-risk categories was published by the IOF and the ESCEO [37]. Basically, this was based on the assessment of the 10-year probability of a major osteoporotic fracture (clinical spine, hip, forearm, or humerus). Women with fracture probabilities below the lower assessment threshold can be considered at low risk. Women with probabilities above the upper assessment threshold can be considered for treatment. Women with probabilities between the upper and lower assessment threshold should be referred for BMD measurements and their fracture probability reassessed. The subgroup eligible for treatment was then stratified into high- and very high-fracture risk categories as will be shown below.

This new concept of high-fracture risk was driven by the data emerging from drug trials of the recently approved romosozumab, abaloparatide, and the established medications such as teriparatide. In contrast to anti-resorptive therapies, anabolic agents demonstrated a more rapid and greater fracture risk reductions [31, 38, 39]. Such strategy of tailoring the medical management to the 
patient's needs represents a revolution in the management of osteoporosis, particularly for those subjects at very high-fracture risk. So, whilst the current guidelines for management of post-menopausal women at highfracture risk advise to start with anti-resorptive therapy (mostly oral bisphosphonates) [22, 40, 41], according to the recent recommendations, it would be more suitable for post-menopausal women at very high-fracture risk to start treatment with anabolic therapy followed by an anti-resorptive agent [31, 42-44].

\section{Assessment and interventional thresholds}

Two approaches have been published describing how to identify the high- and very high-fracture risk categories; these are the following:

\section{National Osteoporosis Guideline Group (NOGG)}

NOGG developed age-dependent assessment thresholds for the UK. The intervention threshold is set at a risk equivalent to that associated with a prior fracture. Two bounds around the intervention threshold have been identified where the assessment of BMD will help to determine whether the individual close to the threshold either exceed that bound or lie below the intervention threshold. These are called assessment threshold for bones. The lower assessment threshold was set to rule out the requirement for BMD testing among women without any clinical risk factors $[45,46]$. The upper assessment threshold was set at 1.2 times the intervention threshold [47]. Very high risk is identified as the risk lying above the upper assessment threshold, whereas high risk lies between the intervention threshold and the upper assessment threshold. On the other hand, low risk is reported when the risk lies below the intervention

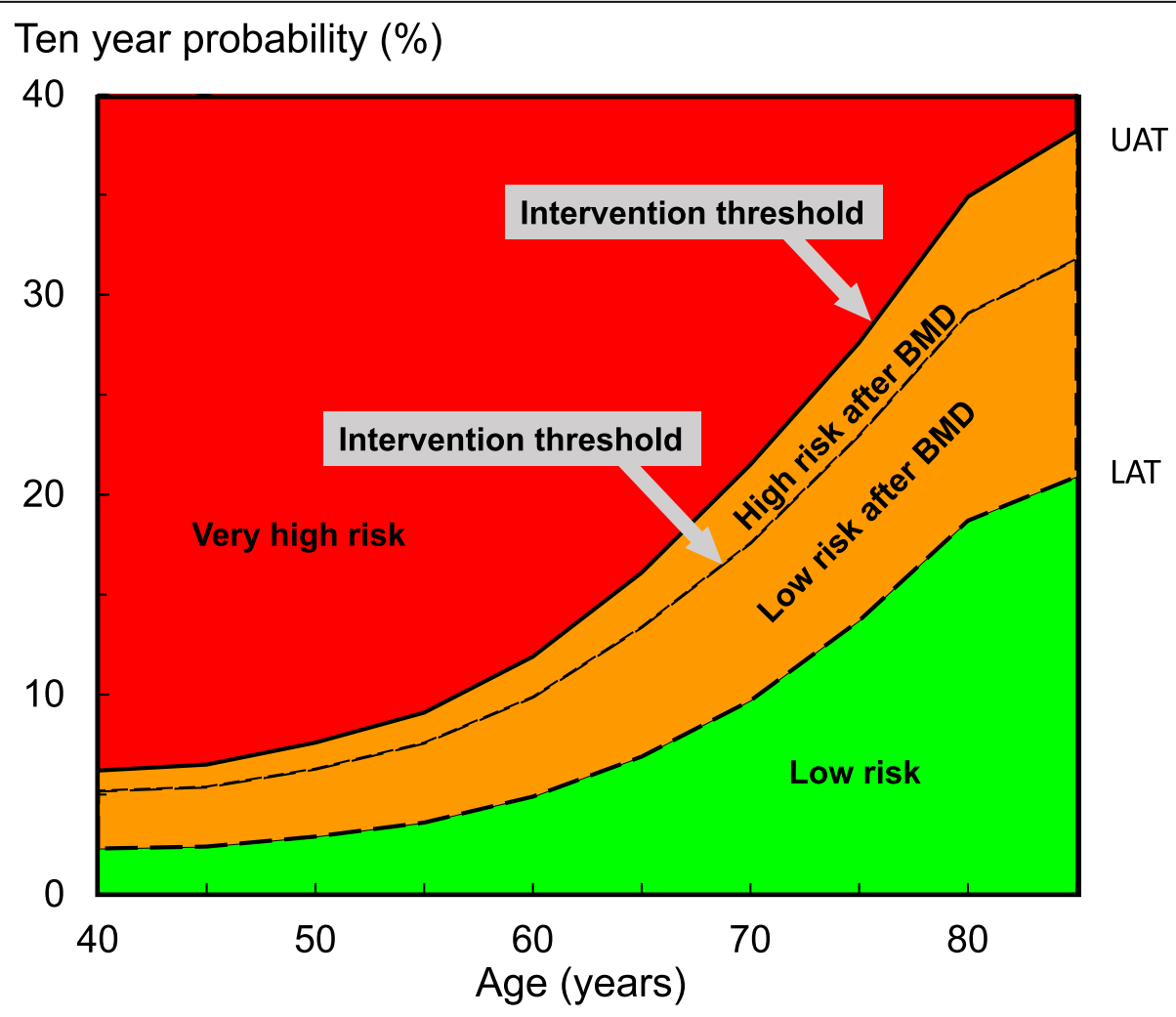

Fig. 1 Infographic outlining the four osteoporosis risk categories. Initial risk assessment relies on using FRAX with clinical risk factors alone. Two intervention thresholds are identified. FRAX probability in the red zone, above intervention threshold (2) indicates very high risk. For this group of people, an initial course of anabolic therapy followed by anti-resorptive treatment may be appropriate. FRAX probability in the green zone suggests low risk, with advice to be given regarding lifestyle, calcium, and vitamin D supplementation. FRAX probability in the intermediate (yellow) zone should be followed by BMD assessment and recalculation of FRAX probability including femoral neck BMD. After recalculation, if the risk got in the red zone above intervention threshold 2, this indicates very high-fracture risk, whereas if the risk got in between intervention threshold 1 and below intervention threshold 2, this would indicate high risk, which suggests initial anti-resorptive therapy. If the risk lies below the intervention threshold 1, this would indicate low risk (management would be similar to green zone. Patients with a prior fragility fracture are designated either at high risk or possibly at very high risk dependent on the FRAX probability (amended from Fig. 1 published in Kanis, J.A., Harvey, N.C., McCloskey, E. et al. Algorithm for the management of patients at low, high and very high risk of osteoporotic fractures. Osteoporos Int 2020; 31: 1-12 (quoted under open access scheme) 
threshold. The assessment thresholds are illustrated in Fig. 1.

\section{European Society of Endocrinology}

In 2019, the European Society of Endocrinology published its algorithm for the management of postmenopausal osteoporosis [48]. The algorithm was based on the proposal that a determination of fracture risk would include measurement of lumbar spine and hip BMD and inserting the total hip or femoral neck BMD value into the FRAX tool. Using that FRAX algorithm, four risk categories were identified: "low risk" includes no prior hip or spine fractures, a BMD $T$-score at the hip and spine both above 1.0 , and 10-year hip fracture risk $<3 \%$ and 10 -year risk of major osteoporotic fractures < 20\%; "moderate risk" includes no prior hip or spine fractures, a BMD $T$-score at the hip and spine both above -2.5 , or 10-year hip fracture risk $<3 \%$ or risk of major osteoporotic fractures $<20 \%$; "high risk" includes a prior spine or hip fracture, or a BMD $T$-score at the hip or spine of -2.5 or below, or 10year hip fracture risk $\geq 3 \%$, or risk of major osteoporotic fracture risk $\geq 20 \%$; and "very high risk" includes multiple spine fractures and a BMD T-score at the hip or spine of -2.5 or below (Table 1 ).

\section{Impact on patients' treatment}

Most of the current clinical guidelines endorse the standard osteoporosis management protocols advising the use of an anti-resportive agent, mostly a bisphosphonate as an initial course of therapy with denosumab given to those who are intolerant to or who have failed bisphosphonate therapy. Anabolic agents are often reserved for severe cases with high fractures risk or those who have failed other initial therapies. Data revealed from recent clinical trials [31, 49] revealed significant anti-fracture benefits with recently approved romosozumab. Studies using abaloparatide build on our longstanding experience with teriparatide in inducing a positive remodelling balance state $[44,50]$. The importance of consolidating the bone mineral density gains achieved from an anabolic agent by following it with an anti-resorptive was also observed. Emerging data favours the use of osteoanabolic agents as a first line regimen for severe osteoporotic cases followed by an anti-resorptive agent. Consequently, several forms of combination and sequential therapeutic modalities have been assessed with positive outcomes. The recently published re-classification of fracture risk categories and the potential of using osteoanabolic agents for subjects with very high risk of fracture as a first option, change the whole treatment paradigms. On the horizon is the potential for targeted osteoporosis therapy tailored to the patient needs. The goals are not only securing gains in bone mass or bone density measure but also improvements in bone quality and reduced fracture rates with minimal skeletal as well as non-skeletal adverse events. Considering the new re-classification of the fracture risk categories, and the potential of reversing the standard sequence of osteoporosis therapy, which is based on starting treatment with anti-resorptive therapy followed by anabolic therapy; there are some hopes that we can reach a state of cure of osteoporosis. These new models of management will be discussed in the following section.

\section{Sequential therapy}

The availability of different osteoporosis therapy options, with 2 main different mechanisms of action whether anabolic or potent anti-resorptive raised the question which treatment modality is the best for the patient and which medication to start treatment with. Clinical trial data show significant anti-fracture benefits with recently approved romosozumab. The extension of FRAME study investigated the efficacy of 1-year treatment with romosozumab followed by 2 years of denosumab [49]. Results revealed further increase in the BMD after switching romosozumab to denosumab. At the end of the 36month period, the subjects who received romosozumab followed by denosumab achieved significantly higher BMD increases from baseline compared to the placeboto-denosumab group (lumbar spine 10.6; total hip 5.2\%; femoral neck 4.8\%) [49]. Furthermore, patients who received romosozumab in the first year of the study exhibited significantly higher fracture risk reductions compared with those who received placebo $(66 \%, 27 \%$, and $21 \%$ for vertebral, clinical, and non-vertebral fractures, respectively). In concordance, in the extension of the $\mathrm{ARCH}$ study, postmenopausal women transitioning to alendronate after 1 year of romosozumab maintained

Table 1 Characteristics of the four osteoporosis risk categories identified according to the European Society of Endocrinology

\begin{tabular}{lllll}
\hline & Low risk & Moderate risk & High risk & Very high risk \\
\hline FRAX & Hip: $<3 \%$ & Hip: $<3 \%$ & Hip: $\geq 3 \%$ & Hip: $\geq 3 \%$ \\
& Spine: $<20 \%$ & Spine: $<20 \%$ & Spine: $\geq 20 \%$ & Spine: $\geq 20 \%$ \\
BMD & Above -1.0 & -1.0 to -2.5 & $\leq-2.5$ & $\leq-2.5$ \\
Fracture & No prior hip or spine fractures & No prior hip or spine fractures & A prior hip or spine fractures & Multiple spine fractures \\
\hline
\end{tabular}


the BMD gains at lumbar spine, total hip, and femoral neck BMD, which were initially achieved with romosozumab without further increases [31]. However, over a total period of 24 months treatment with romosozumab followed by alendronate, resulted in a higher fracture risk reduction of $48 \%$ for vertebral fractures, $27 \%$ for clinical fractures, $19 \%$ for non-vertebral fractures, and $38 \%$ for hip fractures compared with alendronate alone [31].

In the DATA-Switch study, 2 years of teriparatide therapy followed by 2 years of denosumab resulted in further increases in the BMD [50]. Results of the study showed that when denosumab is given for 2 years after 2 years of teriparatide, there was additional increase in the spine BMD by $9.4 \%$ (18.3\% total 4 -year increase) and increased total hip BMD an additional 4.8\% (6.6\% total 4-year increase). In other publication of the abaloparatide, in the trial by Bone et al. [44], alendronate was administered after abaloparatide (given for 18 months), which maintained the fracture risk reduction relative to placebo.

In summary, results of these studies using anabolic agents as first treatment modality followed by an antiresorptive agent consolidate the bone mineral density gains achieved from the anabolic agent and impact positively on the fracture risk reduction.

\section{Combination therapy}

Combination therapy refers to co-administration of an osteoanabolic agent (most studies referring to teriparatide) with a variety of anti-resorptive agents or HRT with other anti-resorptives [51]. Among all combination treatments published so far, studies of teriparatide and denosumab co-administration demonstrated the best and most promising results. In the DATA trial, which included a cohort of largely treatment-naïve postmenopausal women, the teriparatide/denosumab combination treatment induced greater increases in all the three sites: lumbar spine, total hip, and femoral neck as well as radius $\mathrm{BMD}$ compared to either agent alone after 12 [52] and 24 months of therapy [53]. BMD changes with the teriparatide/denosumab combination in this study were similar to those seen with the teriparatide/zoledronate combination in the first 6 months [54], although the magnitude does not refer to direct comparison. However, in contrast to the terparatide/zoledronate combination, BMD levels continued to increase with the teriparatide/denosumab combination after the first 6 months, when the waning effect of zoledronate on bone resorption is seen. In the DATA-HD trial, the combination of denosumab with higher teriparatide dose $(40$ $\mu \mathrm{g})$, increased lumbar spine as well as total hip BMD more than the standard teriparatide $20 \mu \mathrm{g} /$ denosumab combination therapy $[55,56]$, further supporting rationale of using this combination in severe osteoporosis such as those with very high-fracture risk.

Regarding the other two currently commercially available osteoanabolic agents, abaloparatide and romosozumab, there are no studies published so far on the co-administration of either drug with an anti-resorptive agent.

To accommodate such new classification of the fracture risk, an updated algorithm for management of postmenopausal osteoporosis has been published recently by the Endocrine Society [57] which involves an updated evidence-based approach to the management of osteoporosis developed to accommodate the high-fracture risk category and the new recently approved medications (e.g. romosozumab).

\section{Conclusion}

It is the dawn of a new era in osteoporosis care. The identification of the new concept of very high-fracture risk, highlight a subgroup of individuals who require special care and facilitates the opening of the anabolic window in osteoporosis management. Starting treatment with an anabolic agent, in individuals at very high-risk of fracture seems most appropriate to promptly reduce the fracture risk. Combination therapy with teriparatide and denosumab or zoledronate has achieved higher BMD gains compared to each agent alone; however, due to the high cost, combination therapy is rarely compensated.

\section{Abbreviations \\ Acknowledgements
No acknowledgement. \\ Author's contributions \\ The author(s) read and approved the final manuscript. \\ Funding \\ Nothing to declare. \\ Availability of data and materials NA. \\ Ethics approval and consent to participate \\ Not applicable (NA) \\ Consent for publication \\ NA. \\ Competing interests \\ Nothing to declare.}

BMD: Bone mineral density; IOF: International Osteoporosis Foundation; ESCEO: European Society for Clinical and Economic Evaluation of Osteoporosis and Osteoarthritis; FRAX: Fracture Risk Assessment

Received: 26 September 2020 Accepted: 16 November 2020

Published online: 28 January 2021

References

1. Kanis JA, Oden A, Johnell O et al (2001) The burden of osteoporotic fractures: a method for setting intervention thresholds. Osteoporos Int 12: 417-427

2. Kanis JA, Borgstrom F, De Laet C, Johansson H et al (2005) Assessment of fracture risk. Osteoporos Int 16:581-589 
3. World Health Organisation Assessment of fracture risk and its application to screening for postmenopausal osteoporosis. WHO Technical Report Series 843. Geneva: WHO; 1994. https://apps.who.int/iris/bitstream/handle/10665/3 9142 WHO_TRS_843.pdf?sequence=1. Accessed 14 Dec 2020

4. Kanis J, McCloskey EV, Johansson $\mathrm{H}$ et al (2008) A reference standard for the description of osteoporosis. Bone 42:467-475

5. Kanis JA, Harvey NC, Johansson $\mathrm{H}$ et al (2020) A decade of FRAX: how has it changed the management of osteoporosis? Aging Clin Exp Res. 32(2):187-196

6. Siris ES, Brenneman SK, Miller PD, Barrett-Connor E, Chen Y, Sherwood LM, Abbott TA (2004) Predictive value of low BMD for 1-year fracture outcomes is similar for postmenopausal women ages 50-64 and 65 and older: results from the National Osteoporosis Risk Assessment (NORA). J Bone Miner Res 19:1215-1220

7. Rubin $\mathrm{KH}$, Rothmann MJ, Holmberg T (2018) Effectiveness of a two-step population-based osteoporosis screening program using FRAX: the randomized Risk-stratified Osteoporosis Strategy Evaluation (ROSE) study. Osteoporos Int 29:567-578

8. Kanis JA, Seeman E, Johnell O et al (2005) The perspective of the International Osteoporosis Foundation on the Official Positions of the International Society for Clinical Densitometry. Osteoporos Int 16:456-459

9. Kanis JA, Johansson $H$, Oden A et al (2010) A meta-analysis of the efficacy of raloxifene on all clinical and vertebral fractures and its dependency on FRAX. Bone 47:729-735

10. Kanis JA, Johansson H, Odén A et al (2011) A meta-analysis of the effect of strontium ranelate on the risk of vertebral and non-vertebral fracture in postmenopausal osteoporosis and the interaction with FRAX ${ }^{\circledR}$. Osteoporos Int 22:2347-2355 (with erratum Osteoporos Int. 22: 2357-2358)

11. Harvey NC, Kanis JA, Odén A et al (2015) FRAX and the effect of teriparatide on vertebral and non-vertebral fracture. Osteoporos Int 26:2677-2684

12. Harvey NC, Kanis JA, Odén A et al (2015) Efficacy of weekly teriparatide does not vary by baseline fracture probability calculated using FRAX. Osteoporos Int 26:2347-2353

13. Kanis JA, Harvey NC, Johansson $\mathrm{H}$ et al (2017) FRAX update. J Clin Densitom 20:360-367

14. Kanis JA, Johnell O, Oden A et al (2000) Risk of hip fracture according to World Health Organization criteria for osteoporosis and osteopenia. Bone 27:585-590

15. Grigorie D, Sucaliuc A, Johansson $\mathrm{H}$ et al (2013) Incidence of hip fracture in Romania and the development of a Romanian FRAX model. Calcif Tissue Int 92:429-436

16. Kanis JA, Harvey NC, Cyrus Cooper C et al (2016) A systematic review of intervention thresholds based on FRAX. A report prepared for the National Osteoporosis Guideline Group and the International Osteoporosis Foundation. Arch Osteoporos 11:25

17. Kanis JA, McCloskey EV, Harvey NC et al (2015) Intervention thresholds and the diagnosis of osteoporosis. J Bone Miner Res 30:1747-1753

18. Johansson H, Azizieh F, Harvey NC et al (2017) FRAX- vs. T-score-based intervention thresholds for osteoporosis. Osteoporos Int 28:3099-3105

19. Kanis JA, Harvey NC, Johansson $\mathrm{H}$ et al (2017) Overview of fracture prediction tools. J Clin Densitom 20:360-367

20. Nguyen ND, Frost SA, Center JR et al (2008) Development of prognostic nomograms for individualizing 5-year and 10-year fracture risks. Osteoporos Int 19:1431-1444

21. Hippisley-Cox J, Coupland C (2012) Derivation and validation of updated QFracture algorithm to predict risk of osteoporotic fracture in primary care in the United Kingdom: prospective open cohort study. BMJ. 344:e3427

22. Kanis JA, Cooper C, Rizzoli R, Reginster J-Y, Scientific Advisory Board of the European Society for Clinical and Economic Aspects of Osteoporosis (ESCEO) and the Committees of Scientific Advisors and National Societies of the International Osteoporosis Foundation (IOF) (2019) European guidance for the diagnosis and management of osteoporosis in postmenopausal women. Osteoporos Int 30:3-44

23. Kanis JA, on behalf of the World Health Organization Scientific Group (2008) Assessment of osteoporosis at the primary healthcare level. Technical Report. WHO Collaborating Centre, University of Sheffield, UK Available at: https://www.sheffield.ac.uk/FRAX/pdfs/WHO_Technical_Report.pdf. Accessed 19 Sept 2020

24. Johnell O, Oden A, Caulin F, Kanis JA (2001) Acute and long-term increase in fracture risk after hospitalization for vertebral fracture. Osteoporos Int 12: 207-214
25. Johnell O, Kanis JA, Oden A, Sernbo I, Redlund-Johnell I, Pettersen C, De Laet C, Jonsson B (2004) Fracture risk following an osteoporotic fracture. Osteoporos Int 15:175-179

26. Nymark T, Lauritsen JM, Ovesen O, Röck ND, Jeune B (2006) Short timeframe from first to second hip fracture in the Funen County Hip Fracture Study. Osteoporos Int 17:1353-1357

27. Giangregorio LM, Leslie WD (2010) Manitoba bone density program. Time since prior fracture is a risk modifier for 10-year osteoporotic fractures. J Bone Miner Res 25:1400-1405

28. van Geel TACM, van Helden S, Geusens PP, Winkens B, Dinant GJ (2009) Clinical subsequent fractures cluster in time after first fractures. Ann Rheum Dis 68:99-102

29. Johansson H, Siggeirsdóttir K, Harvey NC, Odén A, Gudnason V, McCloskey E, Sigurdsson G, Kanis JA (2017) Imminent risk of fracture after fracture. Osteoporos Int 28:775-780

30. Kendler DL, Marin F, Zerbini CAF, Russo LA, Greenspan SL, Zikan V, Bagur A, Malouf-Sierra J, Lakatos P, Fahrleitner-Pammer A, Lespessailles E, Minisola S, Body JJ, Geusens P, Möricke R, López-Romero P (2018) Effects of teriparatide and risedronate on new fractures in post-menopausal women with severe osteoporosis (VERO): a multicentre, double-blind, double dummy, randomised controlled trial. Lancet 391(10117):230-240

31. Saag KG, Petersen J, Brandi ML, Karaplis AC, Lorentzon M, Thomas T, Maddox J, Fan M, Meisner PD, Grauer A (2017) Romosozumab or alendronate for fracture prevention in women with osteoporosis. N Engl J Med 377:1417-1427

32. Barrionuevo P, Kapoor E, Asi N, Alahdab F, Mohammed K, Benkhadra K, Almasri J, Farah W, Sarigianni M, Muthusamy K, Al Nofal A, Haydour Q, Wang Z, Murad MH (2019) Efficacy of pharmacological therapies for the prevention of fractures in postmenopausal women: a network metaanalysis. J Clin Endocrinol Metab 104:1623-1630

33. Lou S, Lv H, Yin P, Li Z, Tang P, Wang Y (2019) Combination therapy with parathyroid hormone analogs and antiresorptive agents for osteoporosis: a systematic review and meta-analysis of randomized controlled trials. Osteoporos Int 30:59-70

34. Díez-Pérez A, Marin F, Eriksen EF, Kendler DL, Krege JH, DelgadoRodríguez M (2019) Effects of teriparatide on hip and upper limb fractures in patients with osteoporosis: a systematic review and metaanalysis. Bone 120:1-8

35. Russow G, Jahn D, Appelt J, Märdian S, Tsitsilonis S, Keller J (2019) Anabolic therapies in osteoporosis and bone regeneration. Int J Mol Sci 20(1):83-100

36. Khosla S, Hofbauer LC (2017) Osteoporosis treatment: recent developments and ongoing challenges. Lancet Diabetes Endocrinol 5:898-907

37. Kanis JA, Harvey NC, McCloskey E et al (2020) Algorithm for the management of patients at low/middle/high risk of osteoporotic fracture: a global perspective. Osteoporos Int 31:1-12

38. Cosman F, Nieves JW, Dempster DW (2017) Treatment sequence matters: anabolic and antiresorptive therapy for osteoporosis. J Bone Miner Res 32: 198-202

39. Kendler DL, Marin F, Zerbini CAF et al (2018) Effects of teriparatide and risedronate on new fractures in post-menopausal women with severe osteoporosis (VERO): a multicentre, double-blind, double-dummy, randomised controlled trial. Lancet 391:230-240 (Erratum Lancet 2018; 392: 2352)

40. Compston J, Cooper A, Cooper C et al (2017) UK clinical guideline for the prevention and treatment of osteoporosis. Arch Osteoporos 12:43

41. National Institute for Health and Care Excellence (NICE) (2017) Bisphosphonates for treating osteoporosis. Technology appraisal guidance, vol 464. National Institute for Health and Care Excellence, London https:// www.nice.org.uk/guidance/ta464 (accessed on 20 Sept 2020)

42. Kanis JA, Rizzoli R, Cooper C et al (2014) Challenges for the development of bone forming agents in Europe. Calcif Tissue Int 94:469-473

43. Leder BZ, Tsai JN, Neer RM et al (2016) Response to therapy with teriparatide, denosumab, or both in postmenopausal women in the DATA (Denosumab and Teriparatide Administration) study randomized controlled trial. J Clin Densitom 19:346-351

44. Bone HG, Cosman F, Miller PD et al (2018) ACTIVExtend: 24 months of alendronate after 18 months of abaloparatide or placebo for postmenopausal osteoporosis. J Clin Endocrinol Metab 103:2949-2957

45. Royal College of Physicians (1999) Osteoporosis: clinical guidelines for the prevention and treatment. RCP, London https://shop.rcplondon.ac.uk/ 
products/osteoporosis-clinical-guidelines-for-prevention-andtreatment?variant $=6634657349$ (Accessed on 20 Sept 2020)

46. Kanis JA, Delmas P, Burckhardt P et al (1997) Guidelines for diagnosis and management of osteoporosis. Osteoporos Int 7:390-406

47. Johansson H, Oden A, Johnell O et al (2004) Optimisation of BMD measurements to identify high risk groups for treatment-a test analysis. J Bone Miner Res 19:906-913

48. Eastell R, Rosen CJ, Black DM, Cheung AM, Murad MH, Shoback D (2019) pharmacological management of osteoporosis in postmenopausal women: an endocrine society* Clinical Practice Guideline. J Clin Endocrinol Metab. 104(5):1595-1622

49. Lewiecki EM, Dinavahi RV, Lazaretti-Castro M et al (2019) One year of romosozumab followed by two years of denosumab maintains fracture risk reductions: results of the FRAME extension study. J Bone Miner Res. 34(3): 419-428

50. Compston JE, McClung M, Leslie W (2019) Osteoporosis. Lancet 393(10169): 364-376

51. Cosman F (2014) Anabolic and antiresorptive therapy for osteoporosis: combination and sequential approaches. Curr Osteoporos Rep. 12(4):385-395

52. Tsai JN, Uihlein AV, Lee H et al (2013) Teriparatide and denosumab, alone or combined, in women with postmenopausal osteoporosis: the DATA study randomised trial. Lancet. 382(9886):50-56

53. Leder BZ, Tsai JN, Uihlein AV et al (2014) Two years of Denosumab and teriparatide administration in postmenopausal women with osteoporosis (The DATA Extension Study): a randomized controlled trial. J Clin Endocrinol Metab. 99(5):1694-1700

54. Cosman F, Eriksen EF, Recknor C et al (2011) Effects of intravenous zoledronic acid plus subcutaneous teriparatide [rhPTH(1-34)] in postmenopausal osteoporosis. J Bone Miner Res. 3:503-511

55. Tsai JN, Lee H, David NL et al (2019) Combination denosumab and high dose teriparatide for postmenopausal osteoporosis (DATA-HD): a randomised, controlled phase 4 trial. Lancet Diabetes Endocrinol. 7(10): 767-775

56. Ramchand SK, David NL, Leder BZ et al (2020) Bone mineral density response with denosumab in combination with standard or high dose teriparatide: the DATA-HD RCT. J Clin Endocrinol Metab 105(3):890-897

57. Shoback D, Rosen CJ, Black DM, Cheung AM, Murad MH, Eastell R (2020) Pharmacological Management of Osteoporosis in Postmenopausal Women: An Endocrine Society Guideline Update. J Clin Endocrinol Metab 1:1595-1622

\section{Publisher's Note}

Springer Nature remains neutral with regard to jurisdictional claims in published maps and institutional affiliations.

\section{Submit your manuscript to a SpringerOpen ${ }^{\circ}$ journal and benefit from:}

- Convenient online submission

- Rigorous peer review

- Open access: articles freely available online

High visibility within the field

- Retaining the copyright to your article

Submit your next manuscript at $\boldsymbol{\nabla}$ springeropen.com 